\title{
Software Engineering: Emerging Goals and Lasting Problems
}

\author{
Carlo Ghezzi \\ Dipartimento di Elettronica e Informazione - Politecnico di Milano, \\ Piazza L. da Vinci 32, I-20133 Milano, Italy \\ carlo.ghezzi@polimi.it
}

Software has been evolving from pre-defined, monolithic, centralized architectures to increasingly decentralized, distributed, dynamically composed federations of components. Software processes have been evolving along similar lines, from pre-specified sequential work- flows to decentralized and multi-organization endeavors. The organizations to which software solutions are targeted have also been evolving from highly structured corporates to agile and networked enterprises. All this is affecting the way software is engineered (i.e., conceived, architected, and produced). New difficult challenges arise, while old fundamental problems are still with us. The talk surveys this evolution and tries to identify achievements, challenges, and research directions. 